\title{
Observing a decline in general school functioning associated with depressive disorders in children - a potential role for teachers?
}

\section{Deborah van der Westhuizen, MB ChB, MMed (Psych), MD \\ Christa Krüger, MB BCh, MMed (Psych), MD Department of Psychiatry, Weskoppies Hospital, Pretoria}

Childhood depressive disorders frequently go unrecognised This study explores how a decline in school functioning loften associated with depressive disorders) can be identified by teachers using an 8-item questionnaire. Trained teachers recognised changes in the school functioning of 165 children aged 10 - 14 years, which were associated with possible depressive disorders ('screen positives') as identified by the Depression Self-Rating Scale (DSRS).

Depressive disorders are common in children. Estimates of the prevalence of depression in children and adolescents vary between $2 \%$ and $44 \%$. $^{1-4}$ However, for a number of reasons these disorders frequently go unrecognised. A child is dependent on the parents' insight for evaluation; the child may be incapable of reporting symptoms, as depression is a private illness; the parents themselves may suffer from a depressive disorder and may be unaware of their child's needs; and teachers may be unaware of the potential implications of a decline in school functioning. Even when a child is referred for assessment, the available screening instruments focus on symptoms rather than on a decline in functioning. Furthermore, no comprehensive research has been done on depressive disorders in South African primary schools owing to inadequate financial and personnel resources in the mental health services devoted specifically to the needs of children.

This paper describes a new approach to the above problems. The novelty lies in the recruitment of resources outside the usual health care system, as well as in the focus on identification of a decline in general school functioning that is often associated with depressive disorders. Indeed, the diagnostic criteria for depressive disorders highlight a change from the previous level of functioning, where the child's depressive symptoms must produce social or academic impairment. This functional impairment associated with depressive disorders extends to involve all areas of a child's psychosocial world, school performance, behaviour, and peer and family relationships. ${ }^{2}$ These functional signs could be thought of as red lights that should alert parents and teachers to refer the child for mental health assessment.

The clinical picture of depressive disorders in children largely depends on this functional impairment, which manifests as various behavioural changes according to age and developmental level. For example, on an interpersonal level primary school children may have no friends or fight with peers, be disobedient, or 'backchat' teachers. On an academic level, the child's school marks may drop, his or her concentration may deteriorate, homework might not be done, and the child might increasingly be absent from school. Furthermore, the child may lose interest in hobbies or sports, and may also be poorly groomed.

The aim of this study was to explore how children's functional impairment can be identified taking into account the shortage of child mental health resources in South Africa.

Researchers are in favour of using multiple informants when assessing children for possible depressive disorders. "It has become apparent that once children enter school, teachers are important informants on their functioning and behaviour outside the parental home. ${ }^{7.8}$ Teachers were also found to be more sensitive than parents to internalising (anxiety and depressive) symptoms in children, ${ }^{6}$ and were a valuable source of information on changes in school performance. ${ }^{2}$

Although it is not teachers' primary responsibility to observe internalising symptoms in their learners, we chose teachers as our new resource since they are in an ideal position to observe children objectively over a long period of time and can observe 
a decline in general school functioning as part of their ordinary duties, and such a decline in functioning has been demonstrated to have a link with the internalising disorders such as depression. One problem could be that teachers may not be aware of the potential implication of such a decline in general functioning. Teachers would therefore need awareness training and psycho-education on depression.

\section{Methods and results}

In this study, teachers at two primary schools in Pretoria, Gauteng Province, were trained to recognise behavioural changes associated with depression. The training took the form of workshops, presented by the first author and a registered psychiatric nurse, to the teachers of grades 5 - 7 in each of the two schools. The clinical picture, signs and symptoms of depressive disorders, and the associated decline in general functioning were demonstrated using a lecture, discussions and posters.

The question arose whether standard screening instruments for depression such as a structured interview might be applied in schools. We did not believe that these screening instruments would be appropriate for administration by teachers, who operate in an educational environment and do not have training in mental health issues and scales. We thought it might be better to 'translate' the relevant aspects of depression into educationaltype language with which teachers would be familiar and comfortable.

The teachers were trained in the completion and rating of a questionnaire on general school functioning. This questionnaire, developed by the first author, consisted of a Likert-type scale covering a functional decline during the past 12 months in the following eight areas: academic functioning, concentration, completion of homework, interaction with peer group, interaction with teachers, school attendance, self-care, and sports/hobbies. The Likert scale consisted of five categories. With respect to the first item (academic functioning), a score of 1 translated to 'no drop in average marks during the past year', a score of 2 to a 5 - 10\% drop in average marks, a score of 3 to a 11 - 20\% drop, a score of 4 to a $21-30 \%$ drop, and a score of 5 to a drop of more than $31 \%$. With respect to the other items, two anchor points were provided - 'no change/decline' (a score of 1) and 'a severe change/decline' (a score of 5), phrased according to the content of the item. The mean score was calculated for the eight items.
The teachers completed this questionnaire on the school functioning of 165 children, 85 boys (51.5\%) and 80 girls (48.5\%), from grades 5 - 7 , aged 10 - 14 years (mean age 12 years). Although their home languages were predominantly Afrikaans (65\%), Sotho (17\%) and Tswana (9\%), with the remaining 9\% being Zulu, English, Sepedi, Portuguese, Xhosa and Tsonga, all the children were fluent in English at a level appropriate for their age.

Since self-report measures are commonly used to screen for depression, ${ }^{9}$ a research assistant, blinded to the parallel completion of the general school functioning questionnaire and the child's history, administered the Depression Self-Rating Scale (DSRS), ${ }^{5}$ which has been validated for South African children. ${ }^{10}$ The DSRS, an 18-item scale that measures the degree of depression in children aged between 7 and 13 years, was used to categorise possible depressive disorders from mild to severe and also to identify sub-clinical depression. Children with DSRS scores of 9 or less were categorised as not being depressed, those with scores ranging between 10 and 12 as having a mild depressive disorder, and those with scores of 13 or higher as having a moderate, i.e. clinically significant, depressive disorder.

Kruskal-Wallis one-way analyses of variance were done to obtain a preliminary impression of how the decline in general school functioning as observed by the teachers compared with the defined depressive disorder categories on the self-report scale ('screen positives' and 'screen negatives'). The results are summarised in Tablel. These findings suggested that the primary school teachers were able to recognise changes in the behaviour and school functioning of children that were associated with possible depressive disorders I'screen positives') as identified by the DSRS. The yield of $54 \%$ of the children as potentially depressed (unrealistically high) should be seen against the background that the DSRS is merely a screening tool that alerts the system to the fact that further psychiatric assessment is necessary.

\section{Future directions: multidisciplinary network}

Since this was only a pilot study, further studies are needed. It is a limitation that the general school functioning questionnaire was not compared with another method of evaluating functional impairment. In future studies, this questionnaire on general school functioning needs to undergo reliability and validity 


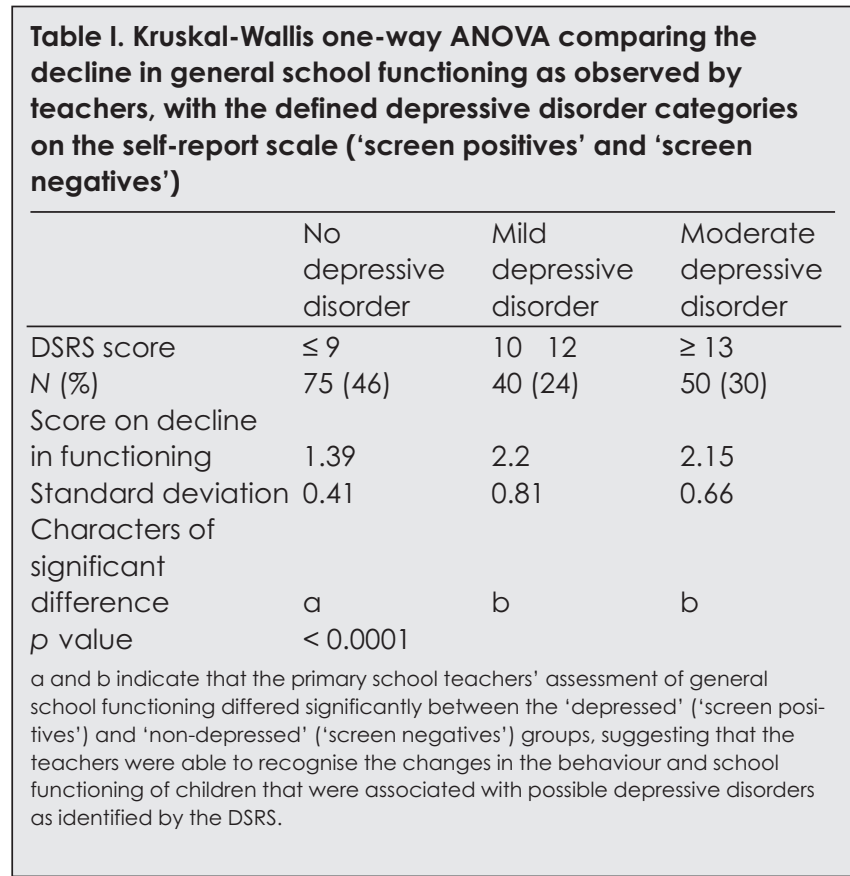

testing (including sensitivity and specificity testing) against a validated and standardised screening questionnaire such as the Strengths and Difficulties Questionnaire (SDQ)." Future studies should also be done to assess whether this questionnaire might be useful as a screening instrument for depressive disorders and comorbid psychiatric disorders in children.

This general school functioning questionnaire is useful in a number of ways. It is more detailed than the functional impairment section of the SDQ and includes more practical examples of functional impairment as found in the school environment. "Although the South African educational system is pressurised, this questionnaire is easy to complete in a short period of time, and we do not envisage that it would place an unacceptable burden on teachers, since evaluation of this sort falls within their ordinary duties. Given the limited availability of child and adolescent mental health services at all levels, this questionnaire might represent a cost-effective way to make such a potentially useful screening assessment accessible to all school-going children without involving mental health professionals.
If this questionnaire is validated, it could serve as a starting point for appropriate referral of children to health services. Ideally, as soon as awareness-trained teachers notice any decline in the child's functioning they could confirm this decline by completing the questionnaire on general school functioning. If alerted by teachers, parents or guardians should then arrange a clinical assessment for the child at the nearest primary for private) health care service.

More broadly, psycho-education and awareness training of parents, teachers and other caregivers such as relatives, afterschool carers and general practitioners should form a part of the ongoing development of a multi-disciplinary team in the community. In this way multi-disciplinary resources would establish a network in the community to ensure prompt referrals. The possible long-term benefit might be that if changes in school functioning associated with depressive and comorbid disorders in children are picked up earlier, complications such as school drop-out, poor relationships, substance abuse, antisocial behaviour and suicide might be prevented or alleviated.

\section{References}

1. Dwivedi KN, Vasma VP. Depression in Children and Adolescents. London: Whurr Publishers, 1997

2. Son SE, Kirchner JT. Depression in children and adolescents. Am Fam Physician 2000; 62 (10): 2297-2308, 231 1-2312

3. Knapp M. Economic evaluations and interventions for children and adolescents with mental heal h problems. J Child Psychol Psychiatry 1997; 38 (1): 3-25.

4. McClure E, Rogeness GA, Thompson NM. Characteristics of adolescent girls with depressive symptoms in a so-called 'normal sample'. J Affect Disord 1997; 42: 187-197.

5. Corcoran K, Fischer J. Measures for Clinical Practice: A Resource Book. London: Free Press, 1987: 391-392

6. Puura K. Children with symptoms of depression - what do adults see? J Child Psychol Psychiatry 1998: 39(4): 577-585.

7. Rutter M. A children's behaviour questionnaire for completion by teachers: Preliminary findings. J Child Psychol Psychiatry 1967; 8: 1-1 1 .

8. Kolvin I, Nicol AR, Leitch I, Macmillan A. Screening schoolchildren for high risk of emotional and educational disorders. BrJ Psychiatry 1977; 131: 192-206.

9. Wicks-Nelson R, Israel AC. Behaviour Disorders of Childhood. 4th ed. New Jersey: Prentice-Hall, 2000: 146-171.

10. Winkler EF. The usefulness of he Depression Self Rating Scale as a screening measure for black South African children. MA (Clin Psychol) thesis, University of Pretoria, 2000.

1 1. Goodman R, Ford T, Simmons H, Gatward R, Meltzer H. Using the Streng hs and Difficulties screen for child psychiatric disorders in a community sample. Br J Psychiatry 2000; 177: 534-539 